\title{
LA PROTECCIÓN DE LA SEGURIDAD PERSONAL EN EL CÓDIGO PENAL*
}

Jean Pierre Matus Acuña**

\section{I}

En un primer intento de sistematización de la parte especial del derecho penal chileno, proponían Politoff, Bustos y Grisolía, ubicar la mayor parte de estos delitos bajo el epígrafe "delitos contra el individuo", que comprendía tres grandes subgrupos: a) "Delitos contra el individuo en sus condiciones físicas" (homicidios, aborto y lesiones); b) "Delitos contra el individuo en sus condiciones ideales" (delitos contra la libertad: coacciones, amenazas, secuestros, etc.; y delitos contra el honor); y c) "Delitos contra el individuo en sus condiciones ideales: delitos contra la propiedad". En este primer intento, se justificaba reunir los delitos "contra el individuo", como un grupo separado de aquéllos dirigidos "contra la sociedad", reputando de "plena validez" en el año 1971 el siguiente "célebre párrafo" de v. Liszt, que reproducen: ${ }^{1}$

"El bien jurídico como objeto de protección jurídica es en última línea, y siempre, la existencia humana en sus diversas manifestaciones. Este es el bien jurídico; esto es, el nervio de todos los demás intereses protegidos jurídicamente. Pero la existencia humana aparece o como existencia del particular o como existencia del particular en la totalidad. Por consiguiente, todos los delitos pueden considerarse como ataques contra los bienes jurídicos del individuo o contra los bienes jurídicos de la comunidad"2

Ponencia leída en una primera versión en el Tercer Seminario Internacional de Política Criminal (14 de noviembre de 2008) organizado por el Centro de Estudios de Derecho Penal de la Universidad de Talca, como resultado del Proyecto Fondecyt N N $^{\circ} 1060410$, "Los nuevos desafíos que las nuevas estructuras sociales imponen al Derecho penal”, dirigido por el Dr. Raúl Carnevali R.

** Profesor Titular de Derecho Penal de la Universidad de Talca. Coreo electrónico: jpmatusa@ utalca.cl

1 Politoff, Sergio; Bustos, Juan; Grisolía, Francisco, Derecho penal chileno. Parte especial, Santiago: Ed. Jurídica de Chile, 1971, pp. 39 y 41-43.

2 Liszt, Franz v., Tratado de Derecho penal (1927), II, p. 2, cit. en Politoff/Bustos/Grisolía, p. 39. 
Veinte años más tarde, en su Parte Especial del Derecho Penal Español, Juan Bustos critica esta clasificación en base a la contraposición individuo-Estado, "ya que toda referencia al individuo es también a la sociedad y viceversa y, por otra parte, una separación tajante entre sociedad y Estado en la actualidad no resulta aceptable desde el punto de vista de la ciencia política", agregando que "tal criterio de ordenación resulta hoy inservible frente a los nuevos requerimientos de órdenes referenciales, especialmente el surgimiento de los llamados bienes jurídicos colectivos". Por lo tanto, propone considerar la sistematización "en relación a un sistema social democrático, que exige la intervención del Estado para atender las necesidades de todos y cada uno de los ciudadanos... no sobre la base de una atomización de la sociedad, sino del todo que implica el sistema". La nueva ordenación propuesta por Bustos distingue entre "los bienes jurídicos que están referidos a las bases de existencia del sistema y aquellos que están en conexión con el funcionamiento del sistema"; y aunque respecto de estos últimos ofrece Bustos una detallada formulación en niveles, respecto de "aquellos referidos a las bases del sistema", reconoce que "son los que tradicionalmente han sido llamados bienes jurídicos individuales", rechazando eso sí dicha denominación "tradicional", por considerar que "no es exacta toda vez que éstos dicen referencias la relaciones microsociales, como es el caso de la vida humana, la salud individual, la libertad, etc.", agregando que "sin ellas no es posible la existencia de ningún sistema social". ${ }^{3}$

\section{II}

No quisiera detenerme aquí en una defensa de una u otra posición sistemática, sino únicamente destacar que en ambas puede apreciarse que la conservación empírica de ciertas condiciones que permiten la existencia de los seres humanos en el ambiente constituido por la agrupación en que se encuentran (lo que permitiría, a su vez, la conservación o existencia de dicho grupo), parece constituir para Bustos el substrato empírico, esto es, las "bases" objetivas sobre las que se cimenta el "sistema social mismo" y su "funcionamiento". La diferenciación entre bienes jurídicos "individuales" y "relaciones microsociales" parece destacar, por una parte, el hecho más bien obvio de que la existencia humana puede describirse mejor como un conjunto de relaciones entre los miembros del grupo en que se desarrolla que como una suma aislada de individuos como, desde antiguo, afirmara Aristóteles.

Pero, por otra parte, entre los "bienes jurídicos individuales" se cuentan tradicionalmente la "propiedad" y el "patrimonio", los cuales no parecen tener otro reflejo empírico que el poder fáctico que un individuo o un grupo de ellos tienen sobre ciertas cosas (con exclusión de terceros individuos del mismo grupo o de otros grupos) o sobre ciertos individuos del grupo

Bustos Ramírez, Juan, Manual de Derecho penal. Parte especial. $2^{a}$ ed. aumentada, corregida y puesta al día, Ariel, Barcelona, 1991, p. 4. 
La protección de la seguridad personal en el Código Penal.

para que, eventualmente, éstos entreguen ciertas cosas por decisión del que tiene ese poder. Sin embargo, la vinculación de estas relaciones de poder fácticas con la interpretación que hacemos de las mismas utilizando el lenguaje del derecho es menos evidente que la constatación empírica de que la existencia natural del individuo depende de manera general, fuertemente en sus primeros años, y más débilmente al irse desarrollando paulatinamente, de la atención de los individuos de su grupo de origen. Así, "el derecho a la vida" (al menos de los individuos suficientes para la subsistencia de la especie en el espacio natural que ocupan) parecería más "natural" que el de "propiedad", que reflejaría no ya ciertas condiciones necesarias para la subsistencia, sino relaciones de poder sobre ciertos objetos e individuos que, como en la utopía marxista, podrían alterarse en sus bases, a discreción de esos mismos individuos, si se organizaran de una manera diferente.

Sin embargo, puestos en una situación básica de disputa por los objetos que sirven a la alimentación (subsistencia) individual, la exclusión de terceros del acceso a esos objetos representa la única vía que garantiza la subsistencia del individuo y su grupo y, en ese sentido, la interpretación que podemos hacer de esa condición fáctica como "derecho de propiedad" parece tan "natural" como la de concebir como un "derecho a la vida" la pretensión de un grupo de "garantizar" de alguna manera la existencia física de los individuos que la componen frente a las agresiones de otros individuos del mismo u otros grupos. No obstante, entre ambas condiciones (o "derechos") cabe apreciar un cierto "orden" fáctico: primero se existe, luego se tiene. Pero una vez puesto el individuo en el mundo, tampoco es posible su subsistencia sin la posibilidad fáctica de disponer (con exclusión de terceros) de los medios necesarios para ello.

\section{III}

La posibilidad que ofrece la sistematización propuesta por Bustos, en orden a distinguir ciertas condiciones fácticas que han de considerarse como las bases o el soporte de la construcción de un "sistema social", asociando esas condiciones a las interpretaciones de las mismas como "derechos" dentro de ese "sistema social", no debe interpretarse como si se afirmara que de la constatación empírica de ciertas condiciones necesarias para la subsistencia del ser humano en la tierra y de los grupos humanos existentes en la misma y los espacios que controlan de ella surgiera o se dedujera el deber de los individuos y grupos humanos de hacer posible tales condiciones o el derecho de tales individuos o grupos a que otros soporten fácticamente las limitaciones que a su propia existencia supone la mantención de las condiciones naturales de existencia de los primeros. Lo que aquí simplemente se quiere destacar es que existen determinadas condiciones y relaciones empíricas que pueden o no interpretarse como lo que denominamos normas jurídicas, pero que de ningún modo están determinadas por esa interpretación y es incluso probable que dichas interpretaciones sean, de cierto modo, una forma imperfecta de describir tales condiciones y relaciones: una que oculta el hecho de que tales condiciones y relaciones no se encuentran fácticamente garantizadas (porque la escasez impone la competencia tanto por la subsistencia como por el control de los medios que la 
hacen posible; porque en grupos extendidos no es necesaria la subsistencia de todos sus individuos para la subsistencia de algunos o la mayor parte de ellos; porque tampoco todos los individuos ni todos los grupos tienen acceso a los mismos medios de subsistencia y defensa de éstos; porque existen individuos más o menos propensos a soportar las cargas de la vida en grupo, etc.).

\section{IV}

Según Kant, a quien difícilmente se le asociaría con ningún tipo de materialismo, en el famoso caso de la Tabla de Carneades, habría un equívoco en la designación de "la necesidad" como "un derecho", pues no sería más que un "supuesto derecho", cuya contradicción con la "Teoría del Derecho" sería evidente ["es fällt in die Augen"], dado que no existiría ningún derecho "objetivo", "prescrito por la ley", para "tomar la vida de otro que no me ha hecho ningún mal", aún "en caso de peligro de perder mi propia vida"; sino sólo una pretensión "subjetiva" que pertenece a la "ética" y que el sentenciador eventualmente "podría llegar a comprender", pero no a justificar. Luego, puesto que "la pena con que amenaza la ley no podría llegar a ser más grave que la pérdida de la vida”, para el náufrago que arrebata la tabla al que la poseía primero, el hecho no sería "tanto como no castigable [unsträflich] (inculpable), sino más bien como no penable [unstrafbar] (impunibile)." ${ }^{4}$

Pocos parecen haber reparado en las dificultades para cualquier concepción idealista de derivar del argumento de Kant las consecuencias usualmente aceptadas, particularmente la de admitir que quien llegó a la tabla primero "tiene el derecho" y, por tanto, el segundo sólo disculpado ya que la "necesidad" no sería más que un "supuesto derecho" en llegar a la tabla:" la constitución del derecho radica sólo en la fuerza del que llega primero. ${ }^{6}$

En efecto, si hemos de suponer que Kant ha aplicado a este ejemplo el "imperativo categórico", esto es juzgando las conductas envueltas según "una máxima”, que pueda valer

$4 \quad$ Kant, Metaphysik der Sitten, p. 235. Nótese, además, que para fundamentar esta distinción, ha utilizado Kant un concepto puramente utilitarista de la pena (que "la pena con que amenaza la ley no podría llegar a ser más grave que la pérdida de la vida”), aparentemente incompatible con su propuesta metafísica absoluta de justificación de la pena; a menos que uno entienda las propuestas de Kant más bien como el origen de una teoría unificadora, antes que una puramente retribucionista (ver Koriath, "Vereinigungstheorien”, pp. 632-634, quien sigue en esto a Höffe). Sin embargo esta nueva valoración de la teoría de Kant, puede considerarse útil sólo como una forma de salvar las contradicciones de dicho filósofo, pues, como señala Roxin, Strafrecht I, p. 71, nota 6, frente a similar intento de Altenhein, "aún cuando esto fuera cierto -lo que las citas [de Kant] contradicen decididamenteno cambia el hecho de de que históricamente la recepción de la teoría de Kant ha desarrollado sus efectos como teoría absoluta".

5 Las consecuencias "dogmáticas" de este razonamiento las he discutido en otro lugar: Matus, Jean Pierre, $L a$ transformación de la teoría del delito en el derecho penal internacional, Barcelona. Atelier, 2008.

$6 \quad$ Küper, Kant und das Brett, pp. 47-55. 
La protección de la seguridad personal en el Código Penal.

en todo momento como "una ley general", ${ }^{7}$ esto significaría que si uno tiene suficiente fuerza como para consolidar una situación de hecho, debiera ejercer esta fuerza e imponerse a los demás, considerando que tal comportamiento es moralmente aceptable (y, además, permite constituir un "derecho"), pues respondería a una "máxima" que puede valer como ley general (y que, como tal, los demás deben respetar). Y viceversa, quienes carecen de dicha fuerza y se ven enfrentados a situaciones de poder, constituidas de hecho, debieran considerar que la "máxima" de su conducta sería respetar tal status quo (y obedecer al que lo impone) como una "regla general" (lo que constituiría, por tanto, un "derecho" del que detenta el poder).

Como lo dice claramente Fletcher, aunque sin expresar un mínimo de extrañeza o crítica ante esta en ningún caso "idealista", sino puramente "conservadora" y acrítica conclusión a que se arriba con este argumento:

"Kant perceived a fundamental difference between a sailor who has occupied tha plank and seeks to defend it against someone trying to take it away [...] the occupier of the plank represents the status quo, his defense of the plank expresses his rights".

De este modo, la interpretación que se hace de la mantención de ciertas condiciones necesarias para la supervivencia como "derechos" no sólo es imperfecta en el sentido de ocultar que con ello la subsistencia no está en modo alguno garantizada, sino también en el sentido de conferirle un valor moral, en el sentido de considerar como suficientemente fundado que sea indiscutiblemente bueno o bueno en sí el mantenimiento del status quo, aunque éste no provea de las condiciones para la existencia y subsistencia del mayor número de los miembros de un grupo humano determinado, sino que las distribuya de manera desigual en el tiempo, según la fuerza que los individuos y los grupos a que pertenecen han podido acumular en el momento y espacio en que se desarrollan.

\section{V}

Por otra parte, la interpretación de la mantención de las condiciones de existencia con el instrumental jurídico, esto es, por medio de descripciones discretas de sucesos aparentemente distinguibles entre sí, sea por el objeto sobre que recaen, por el modo de ejecución o por la alteración fáctica que parecen producir, es imperfecta también desde otro punto de vista: en el razonable afán por "precisar" las descripciones para otorgar "seguridad" acerca de sus consecuencias es posible "crear" distinciones y clasificaciones que terminan por ocultar el

Kant, Grundlegung, p. 421: "Handle nur nach derjenigen Maxime, durch die du zugleich wollen kannst, dass sie ein allgemeines Gesetz werde."

Fletcher, Georg P., The Grammar of Criminal Law, Vol. 1., New York, Oxford University Press, 2007, p. 51. 
objeto que se describe o, como se dice en nuestro ámbito, se valora: una mutilación no sería un ataque a la existencia de la persona sino a su "integridad corporal" por un medio físico; una violación tampoco supondría un atentado contra la existencia de la persona, sino contra su "libertad sexual"; las condiciones de existencia no serían lo afectado por las amenazas de muerte, con independencia de su plausibilidad, sino la "libertad de autodeterminación"; la sustracción de bienes ajenos, uno contra la "propiedad"; y el secuestro de personas, principalmente un ataque a la "libertad personal" y por lo mismo, al igual que los supuestos anteriores, claramente distinguible de la "anulación" total de esas propiedades corporales, de la capacidad fáctica de excluir a terceros del uso de ciertos bienes o de decidir sobre el uso del cuerpo.

Sin embargo, los objetos que se describen o valoran no se presentan ni en la realidad ni en los instrumentos que se utilizan para describirla de forma discreta, sino entrelazada, lo que respecto a las condiciones básicas de la existencia humana puede verse con claridad en el ejemplo extremo de la Tabla de Carneadas: la disputa física por el único medio de salvación puede verse indistintamente como un atentado a la "propiedad" de ese objeto; como una "privación de la libertad" del que, en definitiva queda confinado a valerse con su propio cuerpo; como un ataque a la "salud" de éste, en la medida que no sea necesaria su total aniquilación para resolver la disputa; como un ataque contra "la vida", dado que su aniquilación es más o menos predecible aunque no se produzca de manera inmediata; o como cualquier "combinación" de ellos.

La existencia en el ordenamiento jurídico chileno de reglas que imponen severas penas a la "combinación" de estos ataques (secuestro con homicidio, lesiones y violación, arts. 141 y 142 CP; violación con resultado de muerte, art. 372 bis CP; robos violentos calificados, art. 433 CP), generalizadas en los artículos 82 y 88 del Anteproyecto de 2005, no hacen sino más que reconocer los difusos límites entre las diferentes formas de ataque a las condiciones básicas de la existencia humana y la insuficiencia de las "clasificaciones" discretas que los textos emplean para abarcarlas.

\section{VI}

Por otra parte, dado que las interpretaciones de las condiciones esenciales para la subsistencia humana mediante lo que llamamos "normas" no parece tener como objetivo su clasificación interna, sino el reconocimiento de que un determinado grupo humano espera que, al menos sus miembros entre sí, no alteren las que hasta ese momento les han permitido sobrevivir, ellas producen una distinción más o menos basta de lo que se estima empíricamente tolerable y lo que no, esto es, lo que se "permitiría" o no como "lícito" o "ilícito". Pero, puesto que de este procedimiento resulta también una clasificación, ella está sujeta a la misma limitación de las subclasificaciones analizadas en el apartado anterior: la pretendida discreción en la valoración del objeto se enfrenta a la continuidad empírica en la existencia del objeto valorado 
La protección de la seguridad personal en el Código Penal.

en su extensión espacio-temporal: ello explica las dificultades que se enfrentan en los límites de las interpretaciones normativas de ciertas condiciones existenciales, partiendo por la delimitación de lo que se conoce como fin y principio de la existencia humana, donde la continuidad de la evolución de las especies, por una parte, y de los procesos vitales, por otra, termina por producir ciertas perplejidades a la hora de buscar un correlato empírico a la expresión "ser humano".

Es cierto que contra esta constatación bien puede argumentarse que precisamente evitar tales incertidumbres y perplejidades sería la función de interpretar la realidad desde el punto de vista normativo, estableciendo de este modo, de manera más o menos discrecional y general, los límites que hagan posible la subsistencia de la especie, ahorrándose así los conflictos y daños que se producirían sin esas limitaciones más o menos discrecionales: la reducción de la complejidad mediante su delimitación más o menos discrecional a través de sistemas normativos permitiría a los seres humanos concentrar sus escasos medios en actividades que permitan generar mejores condiciones para su subsistencia.

Esto parecería reflejarse en el hecho de que los mayores grados de complejidad de las sociedades formadas por grupos humanos van acompañados generalmente por un cada vez más complejo "sistema jurídico" y ello parece correlacionarse con mayores grados de bienestar empírico de los individuos miembros de tales grupos, al menos en el nivel de las posibilidades de desarrollo vital y acceso a los medios que lo hacen posible, como sucede en los que ahora llamamos "países desarrollados". Sin embargo, esta aparente correlación entre complejidad normativa y satisfacción de condiciones básicas de subsistencia no significa que la interpretación normativa de dichas condiciones sea capaz por sí misma de transformar la continuidad en discreción, sino simplemente, de reducir esa complejidad del mundo exterior a términos más o menos acotados, en los límites de la continuidad, de la misma manera que los límites en matemáticas reducen la complejidad de las funciones. Pero, en el límite, no es posible, con el instrumental normativo, obtener un resultado discreto de carácter general, pues la discreción no es una propiedad de los objetos a valorar, sino sólo una pretensión de las valoraciones.

Lo anterior se acentúa por el hecho de que las interpretaciones normativas de la realidad usan como instrumento el lenguaje natural, cuya conocida "textura abierta" no es del caso entrar acá a volver a destacar, sino sólo para señalar que, inevitablemente, en algún punto de la interpretación se toma una decisión que establece el punto en que un suceso deja de considerarse "lícito" y pasa a ser "ilícito" (y viceversa). En el ejemplo del "derecho a la vida" o la existencia del ser humano, ello aparece claramente en las decisiones acerca de la "licitud" o no de terminar fácticamente la con existencia de ciertas células y las agrupaciones de ellas a partir de las cuales pueden desarrollarse empíricamente formas de vida que corresponde a la especie humana, y desde cuándo es o no "lícito" retirar un corazón "vivo" a un paciente para trasplantarlo en otro o, dicho de otro modo más directo: cuándo parece (desde el punto de vista de la interpretación normativa) que las condiciones de existencia de un ser humano pueden 
considerarse fácticamente agotadas, para utilizar partes de su cuerpo en el de otro que tenga posibilidades fácticas de participar en la vida en común del grupo.

\section{VII}

Luego, la tan anunciada "normativización" de los "conceptos" que utilizamos para describir ciertos estados de cosas que se consideran indeseables o deseables para conservar las condiciones esenciales de la existencia de la especie humana no resulta de un proceso "voluntarista", sino que es consustancial a la existencia de interpretaciones de la realidad que llamamos "normas jurídicas", dada su propia imperfección y el necesario establecimiento de límites para que ellas sean un instrumento útil a la sobrevivencia de la especie, y no un simple conjunto de especulaciones. Por lo tanto, las limitaciones de las interpretaciones normativas de las condiciones esenciales de la existencia humana, en tanto descripciones de estados de cosas que vale la pena conservar para la conservación de la propia especie, pueden verse del mismo modo que las limitaciones del resto de los instrumentos que utilizamos para reducir la complejidad y sobrevivir: como inevitables hasta cierto punto.

En lo que atañe a la descripción de las condiciones que parecen necesarias para asegurar la existencia de los individuos y del grupo del que son parte, lo anterior se reduce a la necesaria revisión cuidadosa de las descripciones de los sucesos empíricos que atentan contra tales descripciones, procurando, por una parte, reducir en la medida de lo posible la distancia entre la descripción normativa del suceso y su correlato empírico y, por otra, los ámbitos de decisión discrecional del juzgador. Es evidente que este trabajo es el que se ha ido realizado con mayor o menor conciencia y fortuna por las numerosas generaciones que han participado en las elaboraciones de textos normativos hasta hoy en día y que, en los hechos que pueden describirse genéricamente como ataques al cuerpo de las personas con consecuencias más o menos constatables (lesiones, homicidios, secuestros, violaciones y otras agresiones sexuales) parecería que poco se puede avanzar. Sin embargo, parece que existen todavía ámbitos en los cuales es necesario reducir de alguna manera las incertidumbres que genera la distancia entre la interpretación normativa y los acontecimientos que de este modo se pretenden describir o, dicho en términos forenses, la distancia que separa la construcción del tipo penal y la prueba de su realización, particularmente en lo que se refiere a relaciones de causalidad y los estados mentales de quienes intervienen en ellos. En términos generales, ello supone concebir cada requisito de una descripción normativa como partes de una interpretación posible de sucesos que así lo permitan. Y eso sólo es posible si cada uno de esos requisitos tiene un correlato empíricamente contrastable, esto es, un objeto que pueda ser probado e interpretado como tal, según el estado del arte forense en cada momento.

\section{VIII}

Sin embargo, con el establecimiento de ciertas descripciones normativas para calificar 
como no deseados ("ilícitos") ciertos sucesos que afectan las condiciones esenciales de la existencia humana poco se gana para la existencia humana, si ello no es asegurado de la única manera en que es posible asegurar la expectativa de que tales condiciones se conserven: empíricamente. No es necesario detallados estudios de derecho comparado para suponer que en Alemania, Chile, Colombia y México existen interpretaciones normativas para considerar "ilícito" y "punible" dar muerte a otro ser humano o tomar por la fuerza su cuerpo para satisfacer apetitos sexuales propios o ajenos, ni tampoco grandes estudios de sociología para comprobar que, con independencia de las declaraciones de intenciones ("será penado con...") que acompañan dichas descripciones, los grados de seguridad del cumplimiento de la expectiva que tales declaraciones llevan implícitas (los seres humanos en dichos países no serán atacados al punto de ser aniquilados y los que lo hagan serán sancionados) difieren más o menos sustancialmente entre cada uno de esos Estados, y aún en regiones delimitadas de los mismos, los grados de seguridad del cumplimiento de dichas expectativas.

Y es por ello claramente posible un continuo desde el mínimo hasta el máximo posible de seguridad del cumplimiento de las expectativas que se contienen en las interpretaciones normativas de la realidad, que puede expresarse, en cualquier sociedad, en el número de realizaciones de los sucesos que se pretenden evitar y en la probabilidad de que al suceso descrito como indeseable por una interpretación normativa del mismo le siga la sanción que se declara junto a dicha interpretación.

Cuánto de los escasos recursos de que disponemos estamos dispuestos a invertir en reducir el número de "delitos" que afectan las condiciones de existencia de la mayor parte de los miembros de nuestra comunidad y cómo hacerlo efectivamente, para aumentar la seguridad de que es posible nuestra sobrevivencia en la tierra, es algo para lo cual las discusiones acerca de la "normativización" del derecho penal y los límites de las descripciones típicas no tienen respuesta.

Y, sin embargo, como ahora reconoce Jakobs:

"la Ciencia del Derecho, en cuanto ciencia de la constitución normativa de la sociedad $\mathrm{y}$, en concreto, de aquella que realmente tiene lugar y no de una meramente imaginada, no puede pasar por alto -a diferencia de una ciencia pura de las normas- las condiciones necesarias para la realidad del Derecho, sino que debe tenerlas en cuenta. Las normas jurídicas proporcionan orientación, pero sólo si al mismo tiempo se cimentan cognitivamente". ${ }^{9}$

Jakobs, Günther, El derecho penal como disciplina científica, trad. A.v. Weezel, Madrid, Tecnos, 2008, p. 103s, los subrayados son míos, salvo en pura. 
Hace ya casi 250 años Beccaria enfrentó estos problemas desde su formación como economista. ${ }^{10}$ Hace ya 40 Gary Becker reformuló matemáticamente esta aproximación. ${ }^{11}$ A partir de allí se han desarrollado numerosos estudios en busca de respuestas que permitan perfeccionar el modelo económico de análisis del delito. Cualquiera que sea la deriva de estos estudios y de los de la criminología positiva, lo cierto es que si estamos de acuerdo en que la existencia de los individuos de la especie humana, su seguridad personal, es algo valioso y así parece reflejarse en los Códigos penales actuales, no debemos darle la espalda a las herramientas útiles que ellos ofrecen para el análisis y la administración de los recursos que se destinen a garantizarla, pues sin una garantía razonable de nuestra existencia, el derecho que pretende regularla terminará por referirse únicamente a una entelequia "meramente imaginada". por J. Catalá, Barcelona, Bruguera, 1965.

11 Becker, Gary, "Crime and Punishment: An Ecomic Approach", Journal of Political Economy (1968), pp. 169216. 
El principio de subsidiariedad y su papel en la determinación de las competencias sancionatorias de la Unión Europea. Relación con el principio de complementariedad de la Corte Penal Internacional.

\section{EL PRINCIPIO DE SUBSIDIARIEDAD Y SU PAPEL EN LA DETERMINACIÓN DE LAS COMPETENCIAS SANCIONATORIAS DE LA UNIÓN EUROPEA. RELACIÓN CON EL PRINCIPIO DE COMPLEMENTARIEDAD DE LA CORTE PENAL INTERNACIONAL*}

Raúl Carnevali R.**

\section{Cuestiones preliminares}

Si bien la Corte Penal Internacional y la Unión Europea conforman estructuras organizativas que persiguen fines distintos, sí puede decirse que comparten ciertos presupuestos axiológicos comunes que explicarían su creación y posterior desarrollo, y que dicen relación con el reconocimiento y protección de los derechos fundamentales. Asimismo, ambas tienen como hecho histórico vinculante la Segunda Guerra Mundial. En efecto, en el caso de la Unión Europea, si bien con anterioridad al año 1939 hubo propuestas hacia la integración ${ }^{1}$, el camino definitivo comenzó a transitarse tras la última conflagración. Particularmente suele señalarse

* Este Trabajo ha sido realizado dentro del marco del Proyecto Fondecyt N 1080060 titulado "El Principio de complementariedad: un cambio de paradigma en la justicia internacional”. Una versión preliminar se presentó como ponencia en la Conferencia "La relación entre la Corte Penal Internacional y los Estados. El Principio de complementariedad", organizada por la Universidad de Chile el día 5 de marzo de 2009.

** Doctor en Derecho. Profesor Asociado de Derecho Penal y Subdirector del Centro de Estudios de Derecho Penal de la Universidad de Talca. Correo electrónico: rcarnevali@utalca.cl

1 Para Europa la Primera Guerra Mundial dejó como enseñanza que la división sólo la llevaría a desarrollar un papel menor en el concierto mundial, como asimismo ser fuente continua de conflictos. Por ello el Conde Coundenhove-Kalergi propuso la formación de los Estados Unidos de Europa. Por otra parte, el Ministro francés Briand planteó en 1929, en el seno de la Sociedad de Naciones, la necesidad de crear una Unión Europea. De esta forma el propósito integrador dejó de desarrollarse en el plano meramente teórico, para dirigirse a uno práctico, el que luego sería retomado tras finalizar la Segunda Guerra. En todo caso, con anterioridad pueden mencionarse los trabajos de Kant con su obra La pazperpetua, Montesquieu, Saint-Pierre o Saint-Simon, quienes también formularon ideas dirigidas a una integración. Cfr. Truyol y Serra, Antonio. La integración europea. Idea y realidad. Madrid, 1972, p. 18 y ss.; Béroud, Sophie/Weydert, Jean, Le devenir de l'Europe. Paris, 1997, p. 41-46; Sainte Lorette, Lucien de, La integración europea. Barcelona, 1957, p. 23-24. 
como punto inicial el discurso pronunciado por Churchill en Zurich en $1946^{2}$. No cabe duda que el éxito del proceso integrador europeo radica en que los países que la conforman comparten un horizonte valorativo común, aunque coexistan diversas realidades culturales _como afirma Morin Europa es una unitas multiplex ${ }^{3}$. En este sentido, la construcción del sistema comunitario se apoya en el reconocimiento de los derechos fundamentales y libertades públicas, tal como se aprecia en el Art. 6 del Tratado de la Unión Europea ${ }^{4}$. Este consenso valorativo mínimo expresado en el Tratado pone de manifiesto, por una parte, la vocación democrática y de respeto a los derechos fundamentales que inspiran a los Estados miembros _además, todos forman parte del Consejo de Europa_ y por la otra, la exigencia que deben alcanzar quienes pretenden integrar la Unión ${ }^{5}$.

En lo que respecta a la Corte Penal Internacional también puede decirse que la Segunda Guerra Mundial y sus trágicas consecuencias constituye el paso fundamental hacia su conformación. Si bien es cierto, debieron pasar más de cincuenta años para llegar al Estatuto de Roma, fueron los juicios de Nuremberg los que resultaron determinantes acerca de la necesidad de instaurar una corte internacional que juzgue crímenes internacionales ${ }^{6}$. Precisamente, a través de la resolución número 177 (II) de 21 de noviembre de $1947^{7}$, la

2 Truyol y Serra, La integración, cit. nota n. 1, p. 31. En p. 92-95 se reproduce su discurso. También en http:// www.coe.int/T/E/Com/About Coe/DiscoursChurchill.asp (consultado el 21 de enero de 2009).

Morin, Edgar, Pensar Europa. La metamorfosis de Europa. (trad. B. Anastasi de Lonné), 2 a ed., Barcelona, 1994, p. 63, señala que son las fuentes judías, cristianas, griegas y latinas las que han confluido para formar una síntesis armoniosa que constituyen el substrato específico y el común denominador cultural de Europa.

4 "Artículo 6: 1. La Unión se basa en los principios de libertad, democracia, respeto de los derechos humanos y de las libertades fundamentales y el Estado de Derecho, principios que son comunes a los Estados miembros.

2. La Unión respetará los derechos fundamentales tal y como se garantizan en el Convenio Europeo para la Protección de los Derechos Humanos y de las Libertades Fundamentales firmado en Roma el 4 de noviembre de 1950, y tal y como resultan de las tradiciones constitucionales comunes a los Estados miembros como principios generales del Derecho comunitario.

3. La Unión respetará la identidad nacional de sus Estados miembros.

4. La Unión se dotará de los medios necesarios para alcanzar sus objetivos y para llevar a cabo sus políticas". Así también se reconoce en el proyecto Tratado por el que se establece una Constitución Europea, parte II. En: http://eur-lex.europa.eu/JOHtml.do?uri=OJ:C:2004:310:SOM:ES:HTML (consultado el 21 de enero de 2009). Granado Hijelmo, Ignacio, "Constitución europea y jurisprudencia de sistemas", en Revista de Estudios Europeos. № 9, 1995, p. 41, señala que desde 1964 el Parlamento Europeo ha hecho suyo el informe Birkelback, que estableció como doctrina política de admisión a la Comunidad Europea la exclusión de aquellos Estados cuyos gobiernos carecen de legitimidad democrática y cuyos pueblos no participan en las decisiones del gobierno.

$6 \quad$ En todo caso, después de la Primera Guerra Mundial se comenzó a trabajar en un Estatuto para juzgar crímenes de orden internacional. El propio Tratado de Versalles, en el Art. 227, ordenó el establecimiento de un Tribunal para juzgar al Kaiser Guillermo II. Cfr. Cassese, Antonio, International Criminal Law. ${ }^{\circ}$ ed., New York, 2008, p. 27-31; Schabas, William, An Introdution to the International Criminal Court. $3^{\circ}$ ed. New York, 2007, p. 3; Carnevali Rodríguez, Raúl, "Hacia la conformación de un Tribunal penal internacional. Evolución histórica y desafíos futuros", en Revista de Derecho de la Universidad Católica del Norte. Sede Coquimbo. N 10, 2003, p. 32 y ss.

7 Resolución número 177 (II) de 21 de noviembre de 1947 en http://daccessdds.un.org/doc/RESOLUTION/GEN/ NR0/038/84/IMG/NR003884.pdf?OpenElement (consultado el 21 de enero de 2009). 